\title{
EDITORIAL \\ Task shifting and task sharing for neurosurgeons amidst the COVID-19 pandemic
}

\author{
Faith C. Robertson, MD, MSc, ${ }^{1}$ Laura Lippa, MD, ${ }^{2}$ and Marike L. D. Broekman, MD, PhD, JD ${ }^{3,4}$ \\ 'Department of Neurosurgery, Massachusetts General Hospital, Boston, Massachusetts; ' 2 Department of Neurosurgery, \\ Ospedali Riuniti di Livorno, Italy; ${ }^{3}$ Department of Neurosurgery, Leiden University Medical Center, Leiden; and ${ }^{4}$ Department of \\ Neurosurgery, Haaglanden Medical Center, The Hague, The Netherlands
}

$\mathrm{T}$ He coronavirus disease (COVID-19) pandemic has swept the globe at an unprecedented rate, flooding hospitals and revealing the vulnerabilities of finite supplies and providers. Neurosurgeons have restricted operations to emergency and essential interventions. ${ }^{1}$ Some are being deployed to new intradepartmental roles, others lateralized to provide care for coronavirus patients. The reassignment of staff is a common, often temporary, response to expand coverage in a crisis. However, the coronavirus situation is unique. With the novelty of COVID-19, not even infectious disease and critical care experts are exempt from the learning curve, and individual risk of infection breeds further discomfort. Understandably, these conditions can evoke feelings of fear and powerlessness for patients, families, and providers. In this challenging time, strategic health systems approaches can facilitate timely access to safe and affordable care and transform untoward sentiments into a collective strength.

Task shifting and task sharing are workforce strategies that involve duty redistribution. ${ }^{2}$ Task shifting is transference of clinical autonomy from highly qualified healthcare workers to those with shorter training and fewer qualifications. In contrast, task sharing uses tiered staffing models with collaborative teams of specialists and lessqualified cadres who share clinical responsibility and rely on iterative communication and training to preserve highquality outcomes. The application of task shifting and task sharing for medical, surgical, and neurosurgical specialties in low-resource settings has shown that task sharing is preferred to task shifting to maintain safety. ${ }^{2,3}$ An ideal task-sharing model can be evaluated in 3 phases: training, practice, and maintenance (Fig. 1). ${ }^{4}$

For the first phase-training-the principal step is identifying providers and redistributing in a manner that minimizes "things to be learned" in order to satisfy the "job to be done." One must ensure adequate reserves of those with setting-specific expertise. In the COVID-19 pandemic, the most experienced neurosurgeons are also from the most vulnerable age groups, so their wisdom and skills may be best used via telemedicine encounters, guiding ethical decisions on appropriate neurosurgical interventions, or preserved for neurosurgery-specific cases. Attendings with critical care experience may need to oversee medical ICU care. Residents adept in neurocritical care and placing central lines can undergo intensive skills training to bolster competence and confidence in intubation and ventilator management. Residents staffing neurosurgery services can work remotely when possible to place orders, write notes, call consults, and conduct virtual visits with patients, to provide a buffer in case onsite residents become ill and/or require quarantine. Many centers are assigning "contaminated" and "clean" teams to respective wards. Meanwhile, the reduction in neurosurgical patient censuses permits redeployment of remaining faculty, residents, and advanced-practice providers to support COVID-19-specific care. Hospitals can further benefit from medical student engagement. In the United States, final-year medical students are being issued emergency 90 -day limited licenses for early promotion. ${ }^{5}$ More junior students can assist with supervision. Comprehensively, many can be mobilized.

The subsequent question is what to teach. Training in technical skills requires shorter time intervals than clinical decision-making capacity, so procedures can be offloaded in a task-sharing model with oversight from experienced persons. Throughout Europe, when the gravity of intensive care demand was realized, hospitals halted elective procedures and implemented skills workshops for non-ICU personnel regarding noninvasive ventilation and advanced monitoring, as well as abbreviated courses on relevant care, such as testing guidelines and pulmonary optimization. Many national and international societies have released open-source webinars and podcasts on highyield COVID-19-specific management. Departments, neurosurgery included, may benefit from designating personal protective equipment (PPE) "coaches" to oversee 


\section{Task-Sharing

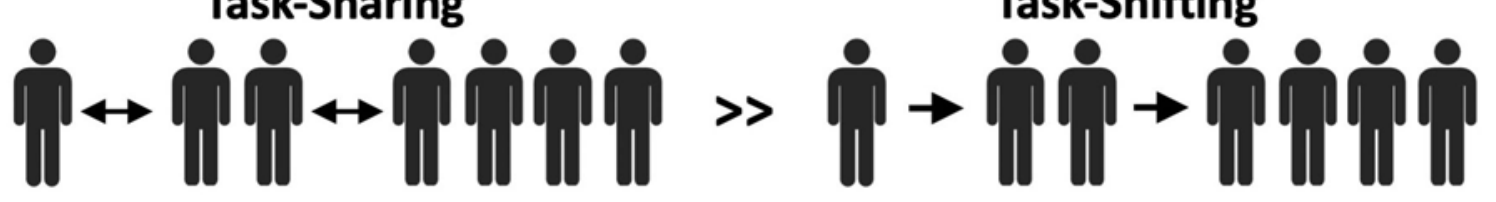

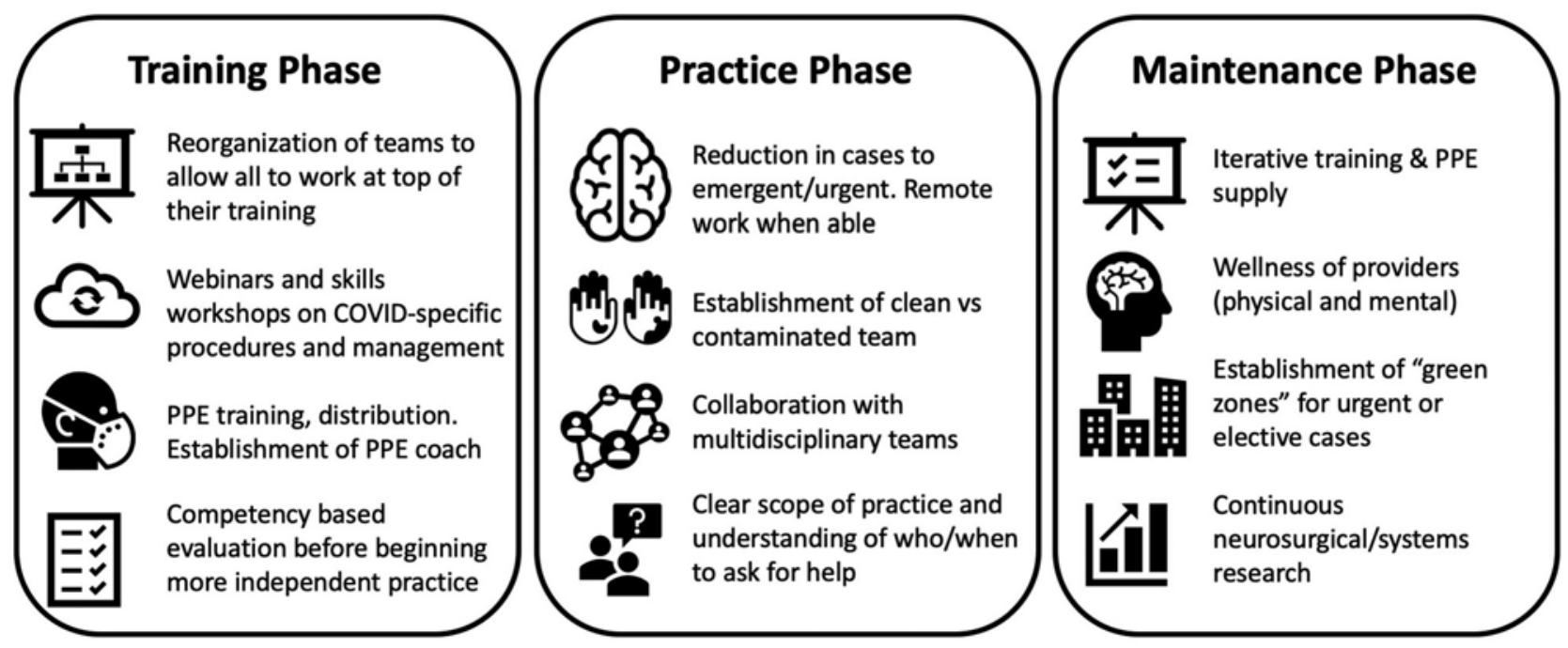

FIG. 1. Task sharing is preferred to task shifting as a method for increasing workforce capacity. An ideal approach involves training, practice, and maintenance phases. Each phase contains its own health system strategies.

proper donning and doffing practices across teams. Concurrent efforts can empower outpatient testing to reduce the burden on hospital centers. Universally, it is essential to ensure adequate governance for standardized curricula, appropriate oversight, and competency-based evaluation before graduating individuals to more independent stages. The escalation of the COVID-19 pandemic means that training phases will be brief, and providers will rapidly advance into the practice phase.

In the practice phase, job scope should be clearly defined to facilitate transitioning into the COVID-19 workforce, seeking consultation for complex cases, and prevention of "task creep," acting beyond permissible guidelines. Within neurosurgical departments, teams can consider dedicating providers for patients at risk for COVID-19 ("contaminated" team). As neurosurgical staff are lateralized to medicine services, we should prepare to humbly engage in working models that involve multidisciplinary teams, supervised in branch models under internal medicine residents and attendings. Again, where to seek help when needed should be clear; preventing task creep protects providers, patients, and the healthcare system. As patient burden exceeds provider capacity, there will be greater pressures to use a task-shifting approach with less oversight, but task sharing is preferred when possible.

The duration of the maintenance phase has yet to be determined. Short-term priorities include ensuring that the newly trained staff thrive for as long as needed, with an adequate supply of PPE and timely dissemination of new information. Addressing the impact of physical, emotion$\mathrm{al}$, and moral stress on providers given isolation and care rationing should be incorporated. The longer-term strat- egy is contingent on multiple factors. If the virus is not seasonal and there is geographic recurrence, control methods may extend into 2022. ${ }^{6}$ If so, our community will need to reassess which cases can truly be delayed. We may need to establish "green zones," as Switzerland neurosurgery is doing, where urgent or elective cases are performed in a separate location, and providers and patients require negative COVID-19 tests and chest radiographs prior to entry. Furthermore, there would be a greater demand for rapid data analysis and iterative systems research to ensure best neurosurgical practices.

While the wrath of coronavirus has caused tremendous disruption in global health already, many cities and countries have yet to experience the virus at its peak. We cannot lie idle, anticipating the storm. In geographic locations currently facing or anticipating the wave of COVID-19 patients, neurosurgeons can begin task sharing to strengthen workforce systems, while continuing to triage operative cases and invest in contingency plans if the pandemic is prolonged. In this arms race between humanity and a virus, we must venture forward with humility, patience, flexibility, and persistence to conquer this challenge together. https://thejns.org/doi/abs/10.3171/2020.4.JNS201056

\section{References}

1. European Association of Neurosurgical Societies. EANS advice: Triaging non-emergent neurosurgical procedures during the COVID-19 outbreak. Accessed April 6, 2020. https://cdn.ymaws.com/www.eans.org/resource/resmgr/ documents/corona/eans_advice2020_corona.pdf

2. Meara JG, Leather AJ, Hagander L, et al. Global Surgery 2030: evidence and solutions for achieving health, welfare, 
and economic development. Lancet. 2015;386(9993):569624.

3. Robertson FC, Esene IN, Kolias AG, et al. Global perspectives on task shifting and task sharing in neurosurgery. World Neurosurg X. 2019:100060.

4. Robertson FC, Briones R, Mekary RA, et al. Task-sharing for emergency neurosurgery: a retrospective cohort study in the Philippines. World Neurosurg X. 2019:100058.

5. Finlaw S. Baker-Polito administration announces emergency actions to address COVID-19. Mass.gov. Accessed April 6, 2020. https://www.mass.gov/news/baker-politoadministration-announces-emergency-actions-to-addresscovid-19

6. Kissler SM, Tedijanto C, Lipsitch M, Grad Y. Social distancing strategies for curbing the COVID-19 epidemic. medRxiv. Published online March 24, 2020. https://www. medrxiv.org/content/10.1101/2020.03.22.20041079v1

\section{Disclosures}

The authors report no conflict of interest

\section{Correspondence}

Faith C. Robertson: frobertson@partners.org.

\section{INCLUDE WHEN CITING}

Published online April 17, 2020; DOI: 10.3171/2020.4.JNS201056. 